\title{
Chondroprotective effects and mechanisms of resveratrol in advanced glycation end products- stimulated chondrocytes
}

Feng-Cheng Liu' ${ }^{1,2}$, Li-Feng Hung ${ }^{3}$, Wan-Lin Wư ${ }^{3}$, Deh-Ming Chang ${ }^{1,2}$, Chuan-Yueh Huang ${ }^{3}$, Jenn-Haung Lai ${ }^{12^{*}}$, Ling-Jun $\mathrm{Ho}^{3,4^{*}}$

\begin{abstract}
Introduction: Accumulation of advanced glycation end products (AGEs) in joints contributes to the pathogenesis of cartilage damage in osteoarthritis $(\mathrm{OA})$. We aim to explore the potential chondroprotective effects of resveratrol on AGEs-stimulated porcine chondrocytes and cartilage explants.
\end{abstract}

Methods: Chondrocytes were isolated from pig joints. Activation of the $\mid \kappa B$ kinase (IKK)-I $\kappa B \alpha$-nuclear factor-kappaB (NF- $\kappa \mathrm{B}$ ) and c-Jun N-terminal kinase (JNK)/extracellular signal-regulated kinase (ERK)-activator protein-1 (AP-1) pathways was assessed by electrophoretic mobility shift assay (EMSA), Western blot and transfection assay. The levels of inducible nitric oxide synthase (iNOS)-NO and cyclooxygenase-2 (COX-2)-prostaglandin $\mathrm{E}_{2}\left(\mathrm{PGE}_{2}\right)$ were measured by Western blot, Griess reaction or ELISA. The expression and enzyme activity of matrix metalloproteinase-13 (MMP-13) were determined by real time RT/PCR and gelatin zymography, respectively.

Results: We show that AGEs-induced expression of iNOS and COX-2 and production of $\mathrm{NO}$ and $\mathrm{PGE}_{2}$ were suppressed by resveratrol. Such effects of resveratrol were likely mediated through inhibiting IKK-I $\kappa \mathrm{B} \alpha-\mathrm{NF}-\kappa \mathrm{B}$ and JNK/ERK-AP-1 signaling pathways induced by AGEs. By targeting these critical signaling pathways, resveratrol decreased AGEs-stimulated expression and activity of MMP-13 and prevented AGEs-mediated destruction of collagen II. Histochemistry analysis further confirms that resveratrol could prevent AGEs-induced degradation of proteoglycan and aggrecan in cartilage explants.

Conclusions: The present study reveals not only the effects and mechanisms regarding how resveratrol may protect cartilage from AGEs-mediated damage but also the potential therapeutic benefit of resveratrol in the treatment of $O A$.

\section{Introduction}

Osteoarthritis (OA) is a highly age-related inflammatory process of joints. Aside from ageing, other factors such as obesity, trauma, genetic predisposition and endocrine factors contribute to the pathophysiological events observed in the process of cartilage and joint damage $[1,2]$. Although age has been recognized as the primary risk factor for the development of OA, the association between ageing and inflammation of the joints remains

\footnotetext{
* Correspondence: haungben@tpts5.seed.net.tw; lingjunho@nhri.org.tw 'Graduate Institute of Medical Science, National Defense Medical Center, Neihu 114, Taipei, Taiwan, ROC

${ }^{3}$ Institute of Cellular and System Medicine, National Health Research Institute, Zhunan, Miaoli County 350, Taiwan, ROC

Full list of author information is available at the end of the article
}

obscure. During the ageing process, there is a phenomenon characterized by post-translational modification of proteins by non-enzymatic glycation and this process results in accumulation of advanced glycation end products (AGEs) in many tissues, including joints $[3,4]$. The accumulated AGEs can reduce proteoglycan and collagen synthesis by chondrocytes and lead to stiffness and fragility of articular cartilage [5]. AGEs also induce the production of matrix metalloproteases (MMPs) that are responsible for causing cartilage degradation and joint damage [6]. In addition to its role in the ageing process, the receptor for AGE (RAGE) appears to play roles in various inflammatory disorders [7]. 
Resveratrol (trans-3, 4',5-trihydroxystilbene), a polyphenolic natural phytoalexin found in a variety of food products, with particularly high levels in grape skin and red wine, preserves many properties such as anti-inflammation, anti-cancer, anti-oxidant and cardio-protection [8-10]. Although limited clinical evidence is available in $\mathrm{OA}$, the anti-inflammatory properties of resveratrol have been shown to suppress anterior cruciate ligament transaction-induced experimental OA in rabbits [11]. Recent studies demonstrate that resveratrol can inhibit interleukin-1 (IL-1)-induced expression of COX-2 and production of $\mathrm{PGE}_{2}$ through suppressing NF- $\kappa \mathrm{B}$ activity in human chondrocytes [12]. Additionally, resveratrol is capable via, in part, its anti-oxidant property of preventing IL-1-induced and p53-induced chondrocyte apoptosis $[13,14]$.

The accumulated studies already suggest the pathogenic roles of cytokines like IL-1 and tumor necrosis factor alpha (TNF- $\alpha$ ) in OA development [15]. Along these cytokines-mediated inflammatory processes, the transcription factors like NF- $\kappa \mathrm{B}$ and AP- 1 have been considered to play critical roles. Regarding this, the results from other researchers and us indicate that comparable to both IL- 1 and TNF- $\alpha$, AGEs can activate NF$\kappa \mathrm{B}$ and AP-1 signaling pathways in chondrocytes $[16,17]$. Furthermore, our results also indicate the critical roles of COX-2-PGE ${ }_{2}$ and iNOS-NO pathways in AGEs-mediated damage of cartilage [17]. In this report, we are interested in determining whether resveratrol can be protective in AGEs-induced inflammation of chondrocytes and damage of cartilage. The results demonstrate that through inhibiting the activation of several critical molecules, resveratrol effectively attenuated AGEs-induced chondrocyte activation and cartilage damage.

\section{Materials and methods \\ Reagents and antibodies}

Polyclonal antisera against iNOS, COX-2 and $\mathrm{I} \kappa \mathrm{B} \alpha$ were purchased from Santa Cruz Biotechnology (Santa Cruz, CA, USA). Polyclonal anti-collagen II antibodies were purchased from Chemicon International (Temecula, CA, USA). The antibodies recognizing ERK, JNK, unphosphorylated IKK $\alpha$ or phospho-IKK $\alpha / \beta$ were purchased from Cell Signaling (Danvers, MA, USA). Resveratrol (R5010) was purchased from Sigma-Aldrich Chemical Company (St. Louis, MO, USA) and was prepared as a $200 \mathrm{mM}$ stock concentration with ethanol. The required concentrations of resveratrol for individual experiments were made by further dilution of the stock preparation with culture medium when needed. Unless otherwise specified, the rest of the reagents were purchased from Sigma-Aldrich Chemical Company.

\section{Preparation of AGEs}

Methylglyoxal-modified albumin, as the source of AGEs examined in this study, was prepared as described [17]. In brief, by incubating $10 \mathrm{mg} / \mathrm{ml}$ of bovine serum albumin (BSA) (USB Corporation, Cleveland, OH, USA) in phosphate-buffered saline (PBS) containing $50 \mathrm{mM}$ methylglyoxal, $0.1 \%$ sodium azide, and $1 \mathrm{mM}$ phenylmethylsulphonyl fluoride (PMSF) at $37^{\circ} \mathrm{C}$ for seven days, the crude preparation of AGEs was obtained. After dialysis against PBS, the AGEs preparation was filtrated and stored at $-80^{\circ} \mathrm{C}$ until use. The AGEs preparation using methylglyoxal-modified albumin contains N(epsilon)-carboxyethyl-lysine (CEL) and other AGEs [18]. To enhance the significance of this study, the AGEs preparation using glycoaldehyde-modified albumin that contains $\mathrm{N}$ (epsilon)-carboxymethyllysine (CML), pentosidine and other AGEs was also prepared according to the report $[19,20]$.

\section{Isolation and culture of porcine chondrocytes}

Porcine cartilage was obtained from the hind leg joints of pigs. The preparation of chondrocytes from cartilage was performed according to our previous report [17]. After enzymatic digestion of articular cartilage with 2 $\mathrm{mg} / \mathrm{ml}$ protease in serum-free Dulbecco's modified Eagle's medium (DMEM) containing antibiotics and 10\% fetal bovine serum (FBS), the specimens were then digested overnight with $2 \mathrm{mg} / \mathrm{ml}$ collagenase I and 0.9 $\mathrm{mg} / \mathrm{ml}$ hyaluronidase in DMEM/antibiotics. The cells were collected, passed through a cell strainer (Beckton Dickinson, Mountain View, CA, USA) and cultured in DMEM containing 10\% FBS and antibiotics for three to four days before use.

\section{Cytotoxicity analysis}

Evaluation of potential cytotoxic effects of resveratral was performed by using 3-(4,-Dimethylthiazol-2-y)-2,5diphenyl-tetrazolium bromide (MTT) colorimetric assays as described [21]. In brief, chondrocytes were incubated in the presence or absence of resveratrol for $48 \mathrm{~h}$. Then, $25 \mu \mathrm{l}$ of MTT $\left(5 \mathrm{mg} / \mathrm{ml}\right.$ in $\left.\mathrm{H}_{2} \mathrm{O}\right)$ was added, and cells were incubated at $37^{\circ} \mathrm{C}$ for $2 \mathrm{~h}$ followed by the addition of $100 \mu \mathrm{l}$ of lysis buffer containing $20 \%$ sodium dodecyl sulfate and $50 \%$ dimethylformamide. After incubation at $37^{\circ} \mathrm{C}$ for another $6 \mathrm{~h}$, the content of dissolved reduced MTT crystals was measured with an ELISA reader (Dynatech, Chantilly, VA, USA).

\section{Transfection and luciferase assays}

Porcine chondrocytes were seeded one day before the transfection experiment at a density of $3 \times 10^{5}$ cells/6cm dish. TransIT ${ }^{\circ}$-LT1 transfection reagent (Pan-vera, Madison, WI, USA) was used to co-transfect $1.5 \mu \mathrm{g} / \mathrm{ml}$ 
of the reporter plasmid pNF- $\kappa \mathrm{B}$-luciferase or pAP-1luciferase with the internal control plasmid pTK-Renillaluciferase (Promega, Madison, WI, USA) at a ratio of 100:1 into cells in serum- and antibiotic-free DMEM. Four hours after transfection, the culture medium was replaced with fresh DMEM supplemented with $2 \%$ FBS. For luciferase assay, total cell lysates were prepared and the firefly luciferase activity was measured and normalized to Renilla luciferase activity according to the dual luciferase reporter system (Promega).

\section{Measurement of $\mathrm{NO}$ and $\mathrm{PGE}_{2}$ concentrations}

The measurement of NO release was reflected by determination of its stable end product, nitrite, in supernatants [17]. The Griess reaction was performed with the concentrations of nitrite measured by a spectrophotometer. In brief, an aliquot $(100 \mu \mathrm{l})$ of culture supernatant was incubated with $50 \mu \mathrm{l}$ of $0.1 \%$ sulfanilamide in $5 \%$ phosphoric acid and $50 \mu \mathrm{l}$ of $0.1 \% \mathrm{~N}$-1-naphthylethylenediamine dihydrochloride. After 10 minutes of incubation at room temperature, the absorbance was measured at $550 \mathrm{~nm}$ wavelength with a plate reader (Tecan, Grodig, Australia). PGE $_{2}$ concentrations were determined by ELISA according to the manufacturer's protocol (Cayman, Ann Arbor, MI, USA).

\section{Western blotting}

ECL Western blotting (Amersham-Pharmacia, Arlington Heights, IL, USA) was performed as described [22]. Briefly, equal amounts of whole cellular extracts were analyzed on $10 \%$ sodium dodecyl sulphate-polyacrylamide gel electrophoresis (SDS-PAGE) and transferred to the nitrocellulose filter. For immunoblotting, the nitrocellulose filter was incubated with Tris-buffered saline with $1 \%$ Triton X-100 containing 5\% non-fat milk for 1 $\mathrm{h}$ and then blotted with antibodies against specific proteins for another $2 \mathrm{~h}$ at room temperature. After washing with milk buffer, the filter was incubated with rabbit anti-goat IgG or goat anti-rabbit IgG conjugated to horseradish peroxidase at a concentration of 1:5000 for 30 minutes. The filter was incubated with the substrate and then exposed to X-ray film (Kodak, Rochester, NY, USA).

\section{Nuclear extract preparation and electrophoretic mobility shift assay (EMSA)}

Nuclear extract preparation and EMSA analysis were performed as detailed in our previous report [22]. The oligonucleotides containing NF- $\kappa \mathrm{B}$-binding site, SP-1 binding site or AP-1-binding site were purchased and used as DNA probes. The DNA probes were radiolabeled with $\left(\gamma_{-}{ }^{32} \mathrm{P}\right)$ ATP using the T4 kinase (Promega). For the binding reaction, the radio-labeled pr)be was incubated with $4 \mu \mathrm{g}$ of nuclear extracts. The binding buffer contained $10 \mathrm{mM}$ Tris- $\mathrm{HCl}(\mathrm{pH} 7.5), 50 \mathrm{mM}$ $\mathrm{NaCl}, 0.5 \mathrm{mM}$ ethylenediaminetetraacetic acid (EDTA), $1 \mathrm{mM}$ dithiothreitol, $1 \mathrm{mM} \mathrm{MgCl} 2,4 \%$ glycerol and $2 \mu \mathrm{g}$ poly $(\mathrm{dI}-\mathrm{dC})$. The reaction mixture was left at room temperature to proceed with binding reaction for 20 minutes. The final reaction mixture was analyzed in a $6 \%$ non-denaturing polyacrylamide gel with $0.5 \times$ Tris/ Borate/EDTA as an electrophoresis buffer.

\section{Gelatin zymography}

Gelatin zymography was performed according to the description by other researchers $[20,23]$. The culture supernatant $(16 \mu \mathrm{l})$ was mixed with $4 \mu \mathrm{l}$ buffer containing 4\% SDS, $0.15 \mathrm{M}$ Tris (pH 6.8), and 20\% glycerol that contains $0.05 \%$ bromophenol blue and analyzed on a $10 \%$ polyacrylamide gel with copolymerized $0.1 \%$ gelatin (Sigma-Aldrich). The positive control of MMP-13 was purchased (EMD Chemicals, Gibbstown, NJ, USA). After electrophoresis, gels were washed with 2.5\% Triton X-100 for three times with 20 minutes each. After incubation with the gelatinase buffer $(50 \mathrm{mM}$ Tris- $\mathrm{HCl}$ (pH 7.6), $10 \mathrm{mM} \mathrm{CaCl}_{2}, 50 \mathrm{mM} \mathrm{NaCl}$, and $0.05 \%$ Brij35 ) at $37^{\circ} \mathrm{C}$ for $24 \mathrm{~h}$, the gel was stained with $0.1 \%$ Coomassie blue, and the clear bands that indicate gelatinolytic activity were visualized under the background of uniform light blue staining. The localization of MMP-13 was identified as judged by the molecular weight of the standards and the report from other researchers [24] under Casio Ex Z60 digital camera (Shibuy-ku, Tokyo, Japan).

\section{Immunoprecipitation kinase assay}

This assay has been described in our previous work [22]. In brief, the whole cellular extract 50 to $100 \mu \mathrm{g}$ was incubated overnight with $5 \mu \mathrm{l}$ of specific antibodies in incubation buffer containing $25 \mathrm{mM}$ HEPES ( $\mathrm{pH}$ 7.7), $300 \mathrm{mM} \mathrm{NaCl}, 1.5 \mathrm{mM} \mathrm{MgCl} 2,0.2 \mathrm{mM}$ EDTA, 0.1\% Triton-X-100, $20 \mathrm{mM} \beta$-glycerophosphate, $0.1 \mathrm{mM}$ Na3VO4, $2 \mu \mathrm{M}$ leupeptin, and $400 \mu \mathrm{M}$ PMSF. The mixture was then immunoprecipitated by the addition of protein A beads and rotated at $4{ }^{\circ} \mathrm{C}$ for $2 \mathrm{~h}$. After extensive wash, the beads were resuspended in $40 \mu \mathrm{l}$ kinase buffer with addition of cold ATP $(30 \mu \mathrm{M}), 8 \mathrm{ng}$ of substrate (GST-c-Jun), and $10 \mu \mathrm{Ci}$ of $(\gamma$-32P)ATP. The mixture was incubated at $30^{\circ} \mathrm{C}$ with occasional gentle mixing for 30 minutes. The reaction was then terminated by resuspending in 1\% SDS solubilizing buffer and boiling for five minutes; then the samples were analyzed in SDS-PAGE.

\section{Real-time RT/PCR}

Total RNA was isolated after lysing the cells by Trizol (Invitrogen, Carlsbad, CA, USA). After reverse transcription of RNA to cDNA, samples were subjected to PCR 
reactions. Real-time quantitation of MMP-13 and GAPDH messenger RNA (mRNA) was performed according to the manufacturer's instructions (CYBR Green Master Mix, Applied BioSystems, Foster City, CA, USA). In brief, $10 \mathrm{ng}$ of cDNA was amplified in a total volume of $20 \mu \mathrm{l}$ consisting of $1 \times$ Master Mix and the gene-specific primers, which were added at a final concentration of $100 \mathrm{n} M$. The primer sequences for both MMP-13 and GAPDH were described by other reports [25,26]: 5'-CCA AAG GCT ACA ACT TGT TTC TTG $-3^{\prime}$ and $5^{\prime}$-TGG GTC CTT GGA GTG GTC AA -3'(for MMP-13); 5'-TGT C AT CCA TGA CAA CTC GG - $3^{\prime}$ and $5^{\prime}$-GCC ACA GTT TCC CAG AGG $-3^{\prime}$ (for GAPDH). The reactions were performed for 50 cycles with $95^{\circ} \mathrm{C}$ for denaturation and $60^{\circ} \mathrm{C}$ for annealing and extension on the ABI 7300 real-time PCR system (Applied BioSystems). The data were collected and the fold changes in gene expression following stimulation in the presence or absence of resveratrol was calculated with the following formula: fold changes =

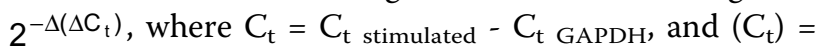
$\mathrm{C}_{\mathrm{t} \text { stimulated }}-\mathrm{C}_{\mathrm{t} \text { control. }}$.

\section{Preparation of cartilage explants}

The preparation of cartilage explants was performed according to our previous report with mild modification [17]. In brief, articular cartilage from the femur head of hind limb joint of pigs was excavated by a stainless-steel dermal-punch $3 \mathrm{~mm}$ in diameter (Aesculap, Tuttlingen, Germany) and weighed. After dissection, each cartilage explant was placed in a 24-well plate and cultured for $24 \mathrm{~h}$ in DMEM containing antibiotics and 10\% FBS. After resting for $72 \mathrm{~h}$ in serum-free DMEM, the cartilage explants were used for further study.

\section{Analysis of cartilage degradation}

Cartilage degradation was assessed by measuring the amount of proteoglycan released into the culture medium as described [17]. In brief, culture medium was added to 1,9-dimethylmethylene blue (DMB) solution (Sigma-Aldrich) in which the metachromatic dye can bind sulfated glycosaminoglycan (GAG), a major component of proteoglycan. The amount of the formation of GAG-DMB complex was measured in a 96-well plate using a plate reader (TECAN, Grödig, Austria) at a wavelength $595 \mathrm{~nm}$. The loss of GAG was calculated and expressed as the total GAG $(\mu \mathrm{g})$ released per $\mathrm{mg}$ (wet weight) of the cartilage weight.

\section{Safranin $\mathrm{O}$ staining and measurement of the aggrecan NITEGE neoepitope}

Cartilage explants were mounted in embedding medium (Miles Laboratories, Naperville, IL, USA) and rapidly frozen at $-80^{\circ} \mathrm{C}$. Serial but incontinuous microscopic sections $(7 \mu \mathrm{m})$ of cartilage explants were cut at $-20^{\circ} \mathrm{C}$ on a Microm cryostat and mounted on Superfrost Plus glass slides (Menzel-Gläser, Braunschweig, Germany). Tissue sections were then stained with Safranin O/fast green countered with Weigert's iron hematoxylin to assess the changes of proteoglycan content [17]. In parallel, the expression of aggrecan NITEGE neoepitope recognized by NITEGE antibodies in tissue sections was determined as described by other researchers [27].

\section{Statistical analysis}

When necessary, the results were expressed as mean \pm SD. Unpaired Student's t-test was used to determine the difference, which was considered to be significant when $P<0.05$.

\section{Results}

Resveratrol inhibited iNOS-NO and COX-2-PGE 2 production in AGEs-stimulated porcine chondrocytes

Because both iNOS-NO and COX-2-PGE 2 pathways are critical in AGEs-induced cartilage damage [17], we determined whether resveratrol could provide any protective effect through regulating these pathways. The AGEs preparation using methylglyoxal-modified albumin that contains CEL and other AGEs was chosen as the stimulant [18]. When chondrocytes were pretreated with various concentrations of resveratrol, the AGEs-induced expression of iNOS was suppressed (Figure 1a). Meanwhile, resveratrol also inhibited AGEs-induced COX-2 expression. The concentrations of $\mathrm{NO}$ and $\mathrm{PGE}_{2}$, both downstream products of iNOS and COX-2, respectively, were measured. The results show that resveratrol inhibited AGEs-induced $\mathrm{NO}$ and $\mathrm{PGE}_{2}$ production (Figure $1 b, c)$. Importantly, resveratrol at a low concentration 25 $\mu \mathrm{M}$ was enough to inhibit $\mathrm{PGE}_{2}$ production (Figure 1c). By both MTT and LDH release assays, we confirmed that at the examined concentrations, resveratrol did not have detectable cytotoxic effects in chondrocytes (Figure $1 d$ and data not shown).

\section{Resveratrol suppressed AGEs-stimulated IKK-I $\kappa$ B $\alpha-\mathrm{NF}-\kappa \mathrm{B}$ signaling}

We next investigated the mechanisms of resveratrolmediated inhibition of iNOS and COX-2 in AGEs-activated chondrocytes. We focused on examining the activity of NF- $\kappa \mathrm{B}$ and AP-1, two families of transcription factors that regulate the activation of iNOS and COX-2 genes and are critical in many inflammatory responses $[28,29]$. Pretreatment with resveratrol resulted in a dosedependent inhibition of AGEs-induced DNA-binding activity of NF- $\kappa$ B (Figure 2a). Because the process of $\mathrm{I} \kappa \mathrm{B} \alpha$ degradation proceeds before NF- $\kappa \mathrm{B}$ activation, we examined whether resveratrol had any effect on $\mathrm{I} \kappa \mathrm{B} \alpha$ degradation. The results show that AGEs treatment 


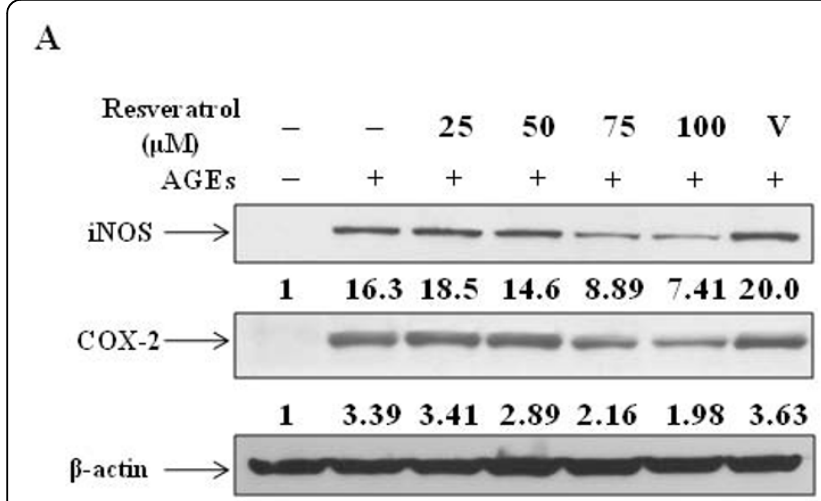

B

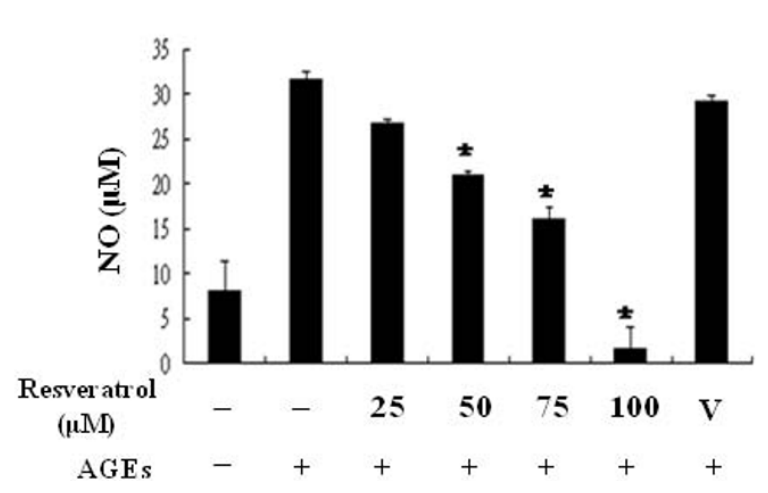

$\mathrm{C}$

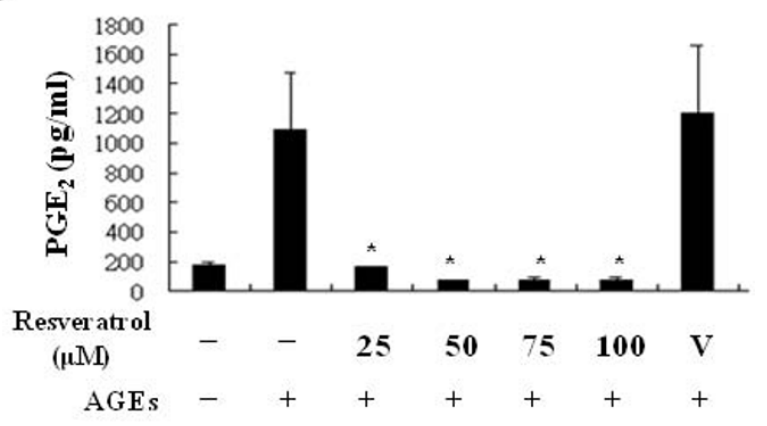

D

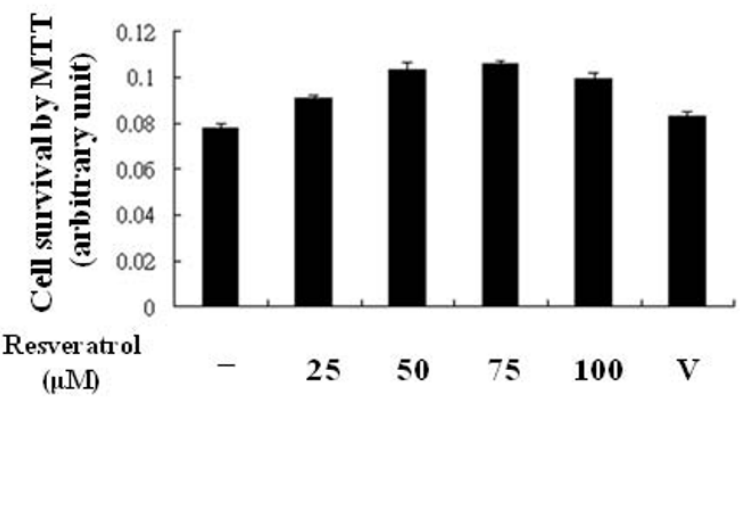

Figure 1 Resveratrol inhibited AGEs-induced expression of iNOS and COX-2 and production of NO and PGE 2 . Chondrocytes $1 \times 10^{6} / \mathrm{ml}$ were pretreated with various doses of resveratrol or the solvent (V), ethanol, for $24 \mathrm{~h}$ and then stimulated with $100 \mu \mathrm{g} / \mathrm{ml} \mathrm{AGEs} \mathrm{for} \mathrm{another} 24$ h. The expressions of iNOS and COX-2 and $\beta$-actin proteins were determined by Western blot (a). The productions of $\mathrm{NO}_{\text {and }} \mathrm{PGE}_{2}$ in the culture supernatants were measured by Griess reactions or ELISA as described in Materials and Methods (b and $\mathbf{c}$ ). Chondrocytes treated with various concentrations of resveratrol for $48 \mathrm{~h}$ were collected and MTT assays were performed to determine possible drug cytotoxicity (d). The values shown in each Western blot represented fold inductions of the densitometric intensity compared to the un-stimulated sample after normalization to internal $\beta$-actin density. The representative data out of three independent experiments are shown. *: $P<0.05$.

caused degradation of $\mathrm{I} \kappa \mathrm{B} \alpha$ with the effect observed $2 \mathrm{~h}$ after treatment (Figure $2 \mathrm{~b}$ ). In the presence of resveratrol, there was dose-dependent reversal of the $\mathrm{I} \kappa \mathrm{B} \alpha$ levels to a certain extent (Figure 2c). Since degradation of $\mathrm{I} \kappa \mathrm{B} \alpha$ requires its phosphorylation by $\mathrm{I} \kappa \mathrm{B}$ kinases, we evaluated whether resveratrol could inhibit IKK $\alpha / \beta$ activities. By Western blotting with antibodies specifically recognizing phosphorylated forms of both IKK $\alpha$ and IKK $\beta$, the results reveal that AGEs effectively induced phosphorylation of IKK $\alpha / \beta$ but had no effect on total IKK $\alpha$ (Figure 2d). In the presence of resveratrol, the levels of AGEs-induced phosphorylated forms of IKK $\alpha / \beta$ decreased (Figure 2e). To determine whether suppression of IKK $\alpha / \beta-\mathrm{I} \kappa \mathrm{B} \alpha-\mathrm{NF}-\kappa \mathrm{B}$ DNA-binding activity could finally lead to decreased transcriptional activation in target genes, luciferase reporter assays were applied. The reporter plasmid pNF- $\kappa \mathrm{B}$-luciferase and the internal control plasmid pTK-Renilla-luciferase were co-transfected into chondrocytes. After transfection, chondrocytes were equally distributed and were stimulated with AGEs in the presence or absence of various doses of resveratrol. Total cell lysates were collected for luciferase activity measurement. We show that consistent with suppression of DNA-binding activity, resveratrol effectively reduced AGEs-induced NF- $\kappa \mathrm{B}$ transcriptional activity in a dose-dependent manner (Figure 2f).

\section{Down-regulation of AGEs-activated AP-1 signaling by resveratrol}

In parallel to its suppressive effects on NF- $\kappa \mathrm{B}$ activation, we demonstrated that resveratrol inhibited AGEsinduced AP-1 DNA-binding activity (Figure 3a). As a comparison, resveratrol did not affect SP-1 DNAbinding activity. Since AGEs can induce activation of both ERK and JNK but not p38 in porcine 


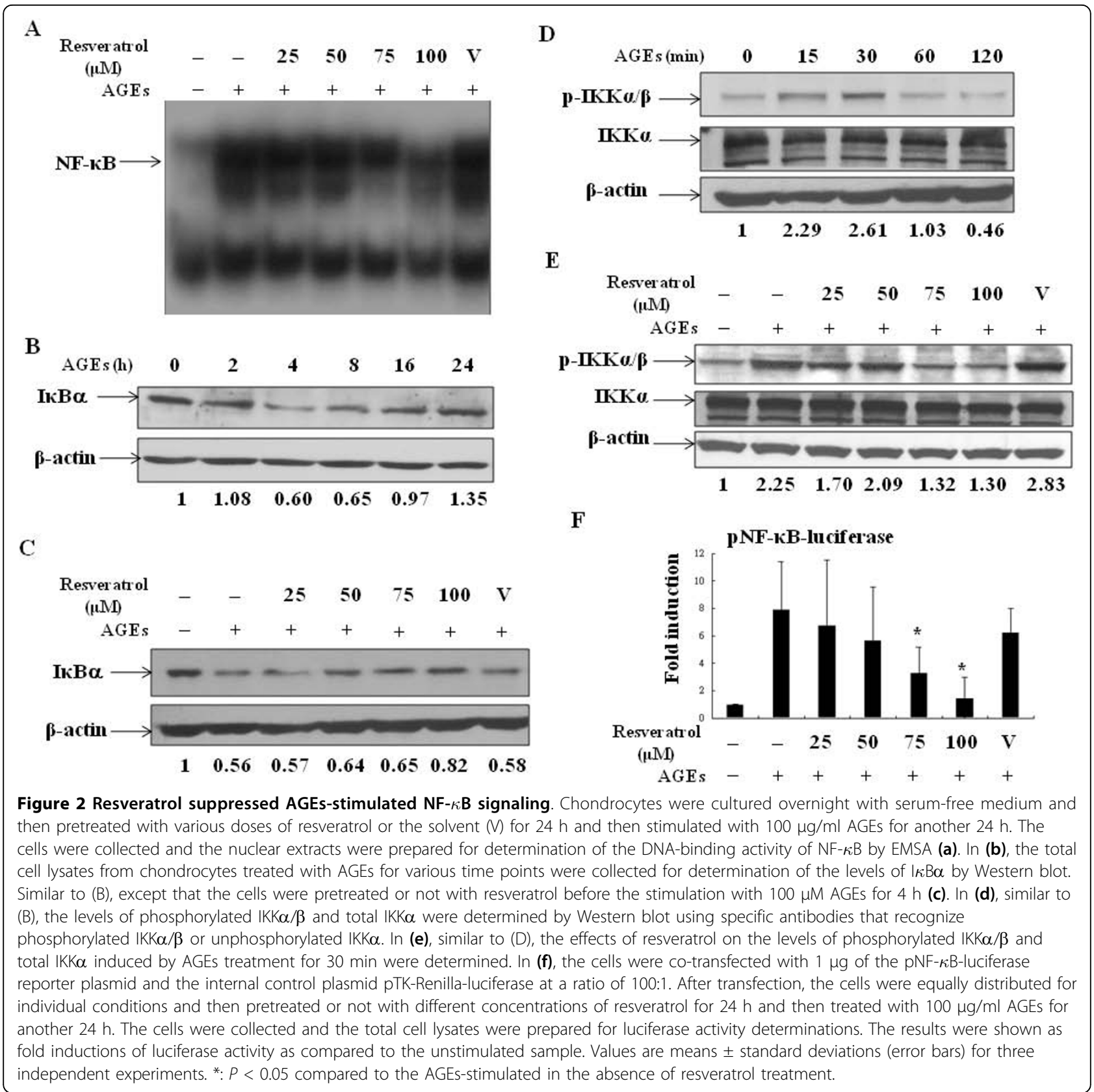

chondrocytes [17], we determined whether resveratrol had any effect on these AP-1 upstream kinase activities. The results show that AGEs-induced phosphorylation of ERK was noticeably suppressed by resveratrol (Figure $3 \mathrm{~b}$ ). In addition, by immunoprecipitation kinase assays, we demonstrated that resveratrol could suppress JNK activity in a dose-dependent manner (Figure 3c); however, resveratrol did not affect the total amount of JNK. Instead, treatment with resveratrol effectively reduced AGEs-stimulated transcriptional activity of AP-1 (Figure 3d).

\section{Resveratrol affected AGEs-regulated MMP-13 expression} and activity and collagen II level

Different from rheumatoid arthritis, in which infiltrating inflammatory cells and synovial pannus are responsible for joint damage, chondrocytes by themselves are primarily the cells causing cartilage damage through generating and secreting cartilage-breakdown enzymes like MMPs. Because MMP-13, regulated by NF- $\kappa \mathrm{B}$ and AP-1 signaling pathways [30], is directly responsible for damaging cartilage matrix, we examined the effects of resveratrol on AGEs-induced MMP-13 activity. As 


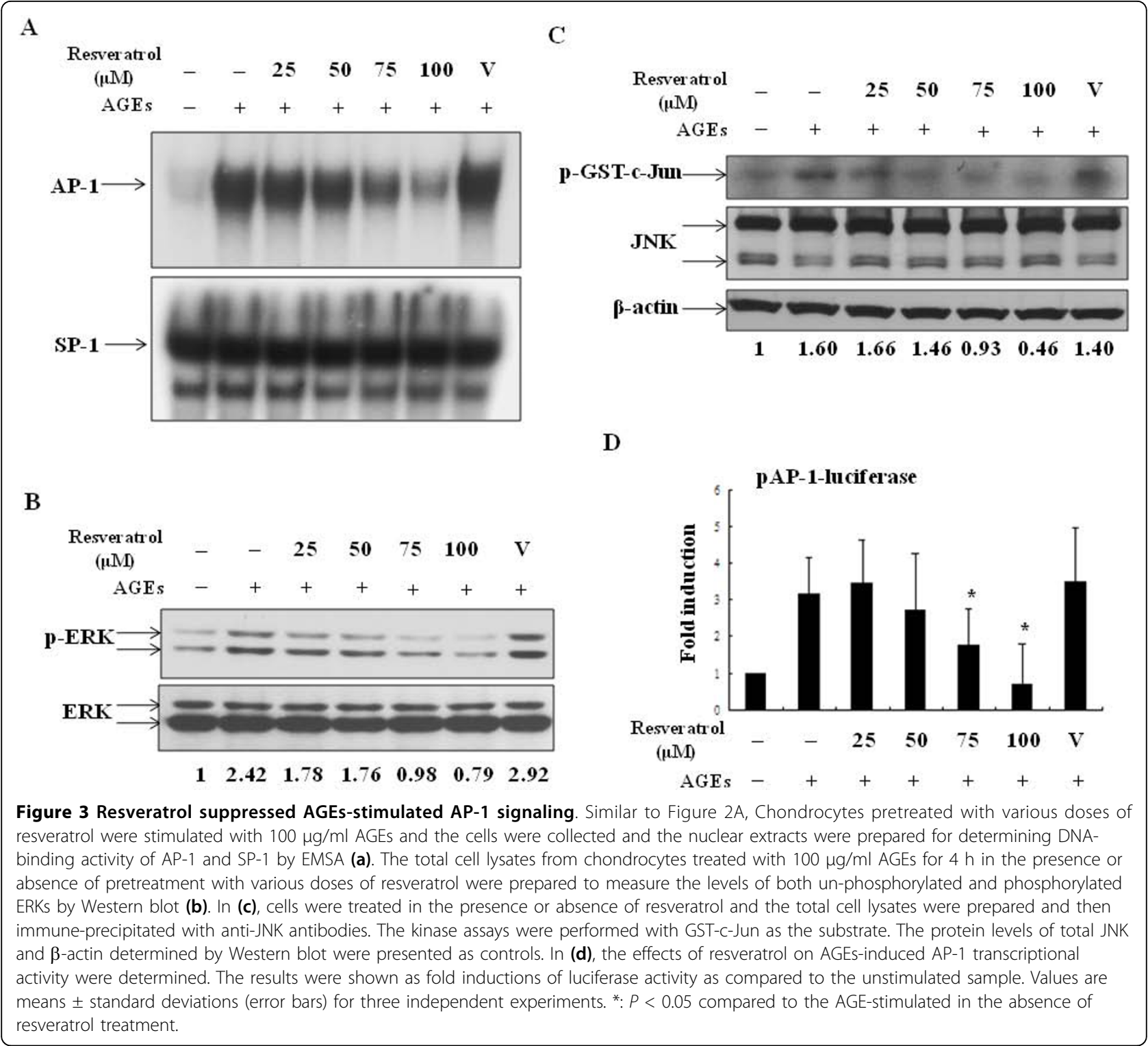

shown in Figure 4a, b, AGEs-induced MMP-13 enzyme activities were suppressed by resveratrol. By quantitative real time RT/PCR analysis, we demonstrate that resveratrol significantly decreased the levels of MMP-13 induced by AGEs treatment (Figure 4c). Since cartilage collagen II is preferentially cleaved by MMP-13, we determined whether resveratrol could affect AGEsmediated destruction of collagen II. As shown in Figure $4 \mathrm{~d}$, resveratrol treatment prevented AGEs-mediated decreases of collagen II by Western blotting. Furthermore, as shown in Additional file 1, resveratrol also suppressed the expression of iNOS and COX-2, the production of $\mathrm{PGE}_{2}$ and $\mathrm{NO}$, the DNA-binding activities of NF- $\kappa \mathrm{B}$ and AP-1 as well as the enzyme activity of MMP-13 in chondrocytes stimulated by another preparation of AGEs using glycoaldehyde-modified albumin that contains CML, pentosidine and other AGEs $[19,20]$. Altogether, the results indicate that resveratrol was able to provide cartilage protection at least by down-regulating AGEs-induced MMP expression and enzyme activity as well as by preventing AGEs-mediated decrease of collagen II in chondrocytes.

Resveratrol protected against AGEs-induced proteoglycan and aggrecan degradation in cartilage explants

To further address the chondroprotective effect of resveratrol as a reflection of its anti-inflammatory property, we investigated whether resveratrol could regulate AGEs-induced degradation of cartilage matrix. When the cartilage explants were stained, a significant 


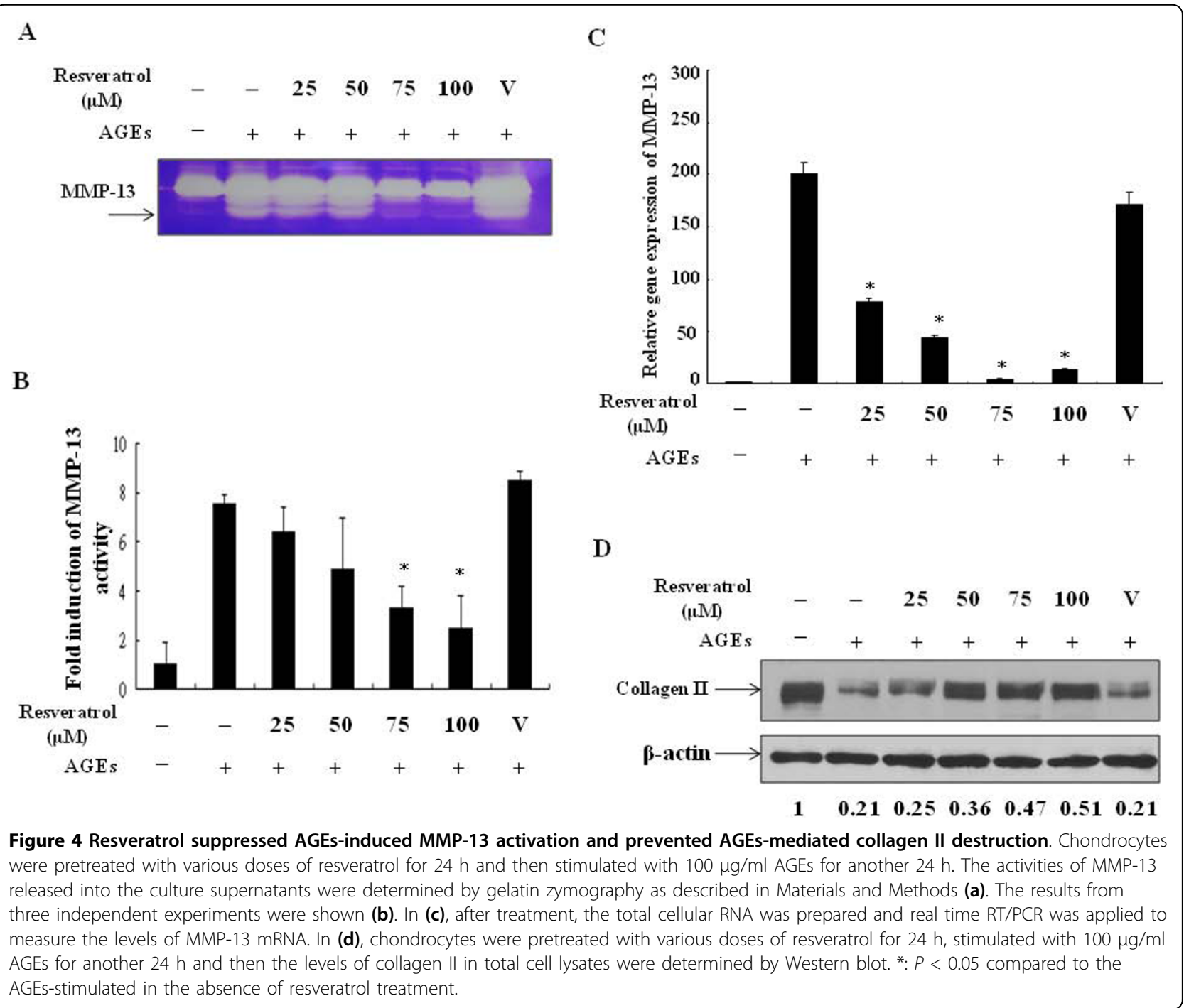

reduction of Safranin O-positive proteoglycan and an increase of cleavage products of aggrecan (NITEGE) became evident in AGEs-treated samples. Such findings were successfully prevented by resveratrol treatment (Figure 5, upper and middle panels). Consistently, we demonstrated that treatment with resveratrol was effective to prevent AGEs-mediated release of proteoglycan into the supernatants of culture of cartilage explants (Figure 5, lower panel).

\section{Discussion}

Aside from pain-control and rehabilitation, there has been no effective therapeutic regimen for joint disorders in OA patients. One of the major reasons is because of OA's characteristics of long-lasting, slow-progression and the varied-intensity of inflammation. Agents with strong immunosuppressive effects are not good choices in considering the potential adverse events from taking such medications. Thus, the drug of choice for OA should be the one that preserves modest anti-inflammatory properties yet has limited or very low toxicity. The components from food or drink consumed in our regular daily activity may be reasonable choices. We therefore, in this study, investigated the potential antiinflammatory action of resveratrol in AGEs-stimulated chondrocytes.

AGEs derived from an array of precursor molecules are very heterogenous in chemical structures. Three major well-characterized AGEs, including pentosidine, CML, and CEL all increase with age in cartilage collagen [31]. Both the sum of these AGEs (CML, CEL and pentosidine) and the AGEs fluorescence correlate significantly with the mean degree of modification of arginine, hydroxylysine and lysine residues in collagen [31]. The concentrations of AGEs products are five-fold higher in elderly OA patients than those in younger patients 


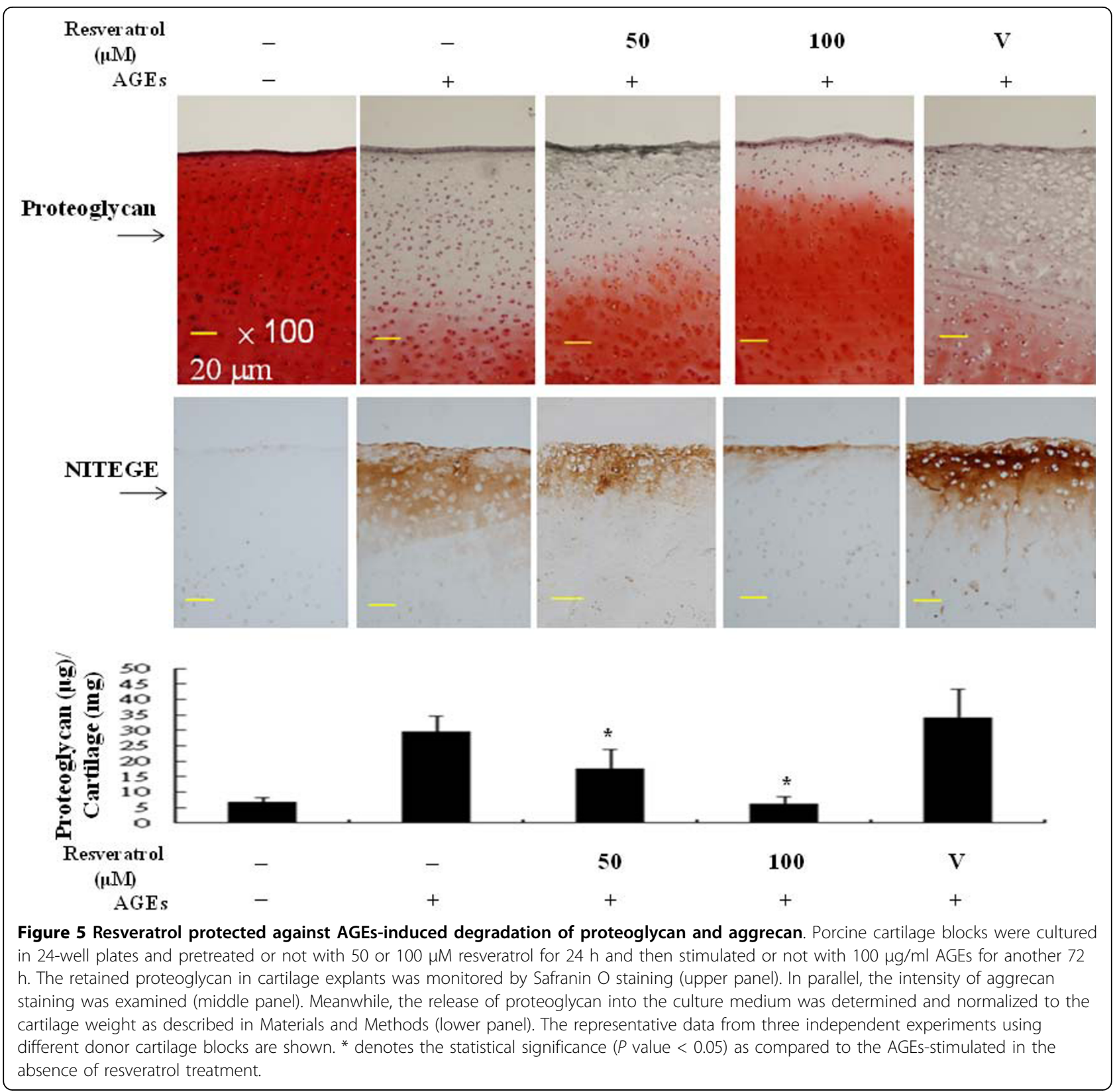

[32,33]. In persons aged 20 to 80 , there is a good linear correlation of increases of fluorescent intensities of accumulated AGEs in cartilage and the difference sometimes can be more than 11-fold higher [31,34]. The fluorescence intensities in AGEs at concentrations ranging from 25 to $100 \mu \mathrm{g} / \mathrm{ml}$ were two- to six-fold higher compared to those in BSA and therefore were close to the conditions in aged joints of human beings (data not shown).

When the physiological concentration of AGEs was examined in this study, the results show that resveratrol could suppress AGEs-induced iNOS-NO and COX-2$\mathrm{PGE}_{2}$ production and the mechanisms were likely through inhibiting IKK-I $\kappa \mathrm{B} \alpha-\mathrm{NF}-\kappa \mathrm{B}$ and JNK/ERK-AP1 signaling pathways. Such anti-inflammatory properties of resveratrol could be faithfully reflected by detecting the degradation of proteoglycan and aggrecan as well as the suppression of proteoglycan release from cartilage explants, the inhibition of collagen II decrease and the attenuation of MMP expression and enzyme activity induced by AGEs (summarized in Figure 6). This study thus provides evidence of chondroprotective effects and mechanisms of resveratrol as well as its potential application in AGEs-mediated pathogenesis in OA joints.

Among the factors contributing to OA pathogenesis, both COX-2 and iNOS play important roles $[35,36]$. 


\section{Advanced glycation end products}

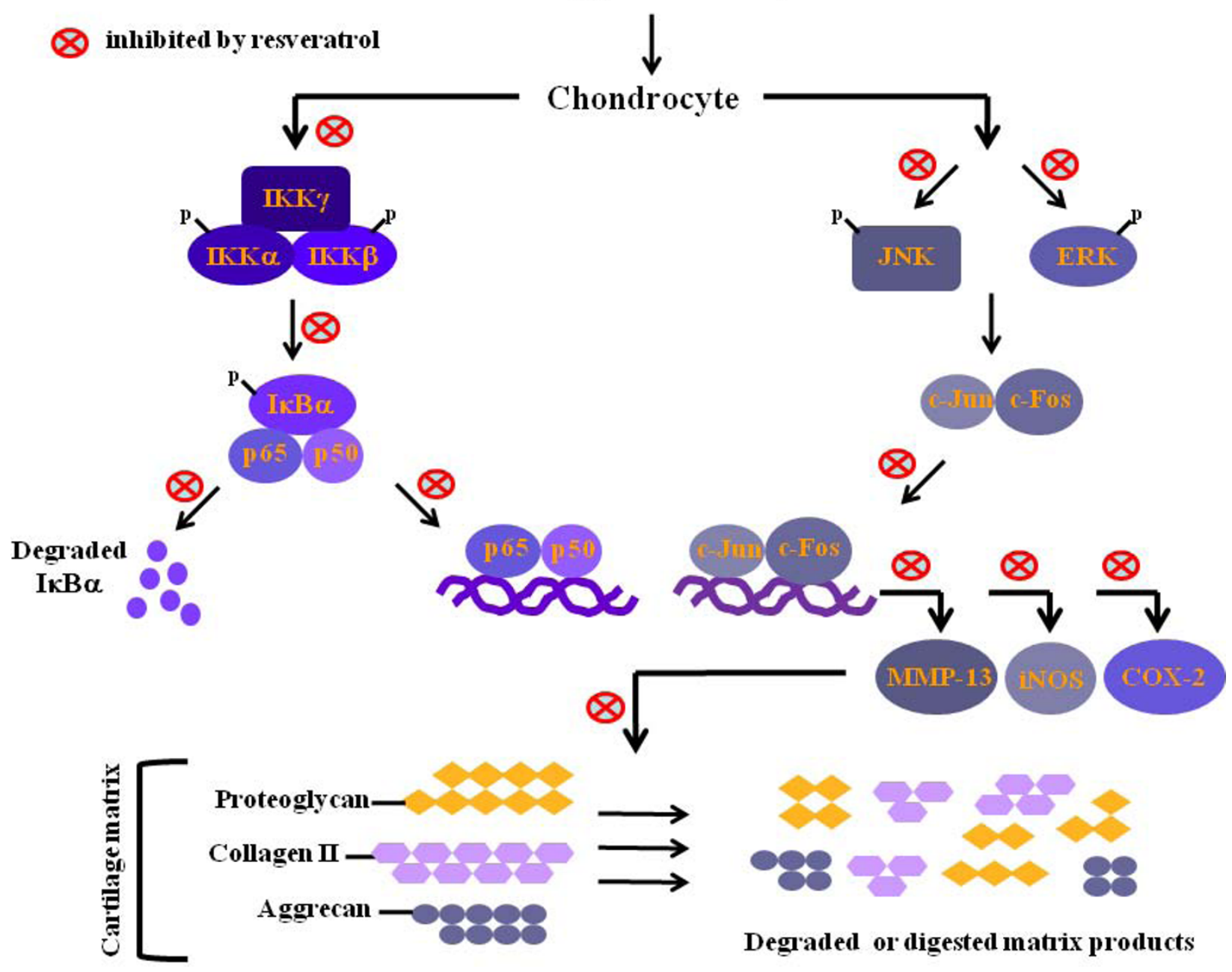

Figure 6 The effects and mechanisms of resveratrol in AGEs-mediated damage of cartilage. AGEs stimulation activated both NF- $\kappa$ B and AP-1 signaling pathways. The activation of NF- $\kappa$ B and AP-1 signaling leads to the generation of iNOS, COX-2 and MMP-13 that cause the degradation of proteoglycan, aggrecan and collagen II, three major components of cartilage matrix, through direct and indirect mechanisms. Along the activation of these critical pathways by AGEs, many associated events were blocked by the treatment of resveratrol as indicated.

Several reports indicate that the inhibition of NO production can suppress IL-1-induced chondrocyte apoptosis and reduce the severity of cartilage damage in experimental OA models $[37,38]$. Given the evidence that activation of the COX-2- $\mathrm{PGE}_{2}$ pathway in chondrocytes results in cell apoptosis, Pelletier et al. [39] showed that the inhibition of COX-2 rescues cells from death in experimental OA of dogs. Treatment with nonsteroidal anti-inflammatory drugs also inhibits IL-1induced MMP-1 and MMP-3 production in bovine articular chondrocytes [40]. The importance of both iNOS and COX-2 in AGEs-mediated pathologies is further supported by our report that successful blockage of either COX-2 or iNOS activity significantly reduces AGEs-mediated proteoglycan release and cartilage damage [17]. The data from Dave et al. [13] also reveal that resveratrol suppresses IL- $1 \beta$-induced COX-2-PGE ${ }_{2}$ activation and mediates anti-apoptosis effects in human articular chondrocytes. Accordingly, the inhibition of both COX-2-PGE 2 and iNOS-NO pathways by resveratrol should suggest that it is a potential chondroprotective regimen.

Consistent with the effects on COX-2 and iNOS, the AGEs-induced DNA-binding and transcriptional activity of NF- $\kappa$ B and AP-1, which also regulate the enhancer/ promoter regions of these two enzymes, were susceptible to the suppression by resveratrol. In addition, the NF- $\kappa \mathrm{B}$ and AP-1 upstream signaling molecules, including IKK$\mathrm{I} \kappa \mathrm{B} \alpha$ and $\mathrm{ERK} / \mathrm{JNK}$, were suppressed by resveratrol. Somewhat different from its effect on IL-1-induced 
upstream molecules regulating NF- $\kappa \mathrm{B}$ activation, reseveratrol suppressed AGEs-induced but not IL-1-induced IKK activity [12]. These observations further distinguish the down-stream signaling events regulated by AGEs stimulation from those induced by the pro-inflammatory cytokine IL-1. Given broad spectrums of NF- $\kappa \mathrm{B}$ - and AP-1-targeted downstream events, resveratrol may exert many more therapeutic benefits in AGEs-mediated pathogenesis other than the evidence demonstrated in this report. In support of this conclusion, resveratrol has been shown to down-regulate the expression of proinflammatory mediator and cytokine genes via inhibiting NF- $\kappa \mathrm{B}$ signaling pathway [14]. The results from this study also highlight the potential of examining small molecule like JNK or ERK inhibitors as suggested by other researchers in ageing-related disorders caused by deposition and accumulation of AGEs [41].

In this study, we further show that aside from the suppression of iNOS-NO, COX-2-PGE ${ }_{2}$, IKK-I $\kappa \mathrm{B} \alpha-\mathrm{NF}-\kappa \mathrm{B}$ and JNK/ERK-AP-1 signaling pathways, resveratrol could potently inhibit the expression and enzyme activity of MMP-13. The results were consistent with earlier studies demonstrating that the activation of MMP-13 critically requires the transcriptional activity of both NF$\kappa \mathrm{B}$ and AP-1 [30]. According to Mitchell et al. [42], MMP-13 expression co-localizes with collagen II degradation in active OA lesions and the inhibition of MMP13 greatly reduces collagen II degradation. In contrast, the increased expression of MMP-13 in cartilages and joints using tetracycline-regulated transcription in conjunction with a cartilage-specific promoter results in exaggerated loss of proteoglycan and cleavage of collagen II in a transgenic animal model [43]. In addition to being the most potent collagen II-degrading enzyme, MMP-13 can degrade proteoglycan and aggrecan and play a dual role in damaging cartilage matrix $[44,45]$. Altogether, the results presented in this report suggest that the treatment with resveratrol helps to protect against AGEs-induced degradation of cartilage components like proteoglycan, aggrecan and collagen II.

There are two major limitations in this study. First, the results of examining porcine chondrocytes could not be exactly translated into those in studying human chondrocytes although the study of choncrocytes from different species has been frequently applied by many researchers. Secondly, the concentrations of resveratrol examined in this study were relatively high. A "good" red wine contains approximately $75 \mu \mathrm{mol} / \mathrm{L}$ content of resveratrol, which makes reasonable wine consumption unlikely to reach effective anti-inflammatory concentrations in plasma in human bodies [46]. Because of its lipophilic character, resveratrol has been shown to accumulate in tissues such as the heart, liver, and kidney [47]. It is therefore concluded that long term absorption of a sufficient quantity of resveratrol may increase the beneficial effect of red wine on health [48]. Indeed, in vitro approaches demonstrate that high concentrations of resveratrol (10 to $100 \mu \mathrm{M})$ have cardioprotective effects by inducing endothelial nitric-oxide synthase gene expression and increasing nitric oxide levels in cultured endothelial cells [46]. High concentrations of resveratrol also inhibit interleukin-5-induced activation of human peripheral blood eosinophils without reduction of cell viability [49]. One of the therapeutic approaches with high concentrations of resveratrol (10 to $100 \mu \mathrm{M})$ in the treatment of AGEs-induced cartilage damage may be performed through local delivery that gains high anti-inflammatory and intra-articular concentrations and provides protection of cartilage from damage by AGEs. Further studies are being performed to investigate this possibility in OA animal models.

\section{Conclusions}

In this study, we show that aside from the suppression of iNOS-NO, COX-2-PGE ${ }_{2}$, IKK-NF- $\kappa$ B and MAPKAP-1 signaling pathways, resveratrol could potently inhibit MMP-13 expression. All these observed effects by resveratrol contributed to the protection against AGEinduced degradation of cartilage components like proteoglycan, aggrecan and collagen II (Figure 6). Understanding the molecular mechanisms activated by accumulated AGEs in joints and how the processes may be modified by resveratrol could provide additional approaches currently unavailable to slow the progression of OA. It is also anticipated that the results from this report will bring more in vitro and in vivo studies to confirm the therapeutic benefits of resveratrol in patients with OA and AGEs-mediated disorders.

\section{Additional material}

Additional file 1: Figure S1. Effects of resveratrol on chondrocytes

activated by an AGEs preparation using glycoaldehyde-modified albumin (gAGEs) that contains $\mathrm{N}$ (epsilon)-carboxymethyllysine $(\mathrm{CML})$, pentosidine and other AGEs.

\section{Abbreviations}

AGEs: advanced glycation end products; AP-1: activator protein-1; BSA: bovine serum albumin; COX-2: cyclooxygenase-2; DMB: 1,9dimethylmethylene blue; DMEM: Dulbecco's modified Eagle's medium; ELISA enzyme-linked immunosorbent assay; EMSA: electrophoretic mobility shift assays; ERK: extracellular signal-regulated kinase; FBS: fetal bovine serum; GAG: glycosaminoglycan; IKK: I $\kappa$ B kinase; IL-1: interleukin-1; iNOS: inducible nitric oxide synthase; JNK: c-Jun N-terminal kinase; MMP: matrix metalloproteinase; MTT: Dimethylthiazol-2-y)-2,5-diphenyl-tetrazolium bromide; NF- $\kappa$ B: nuclear factor-kappaB; OA: osteoarthritis; PBS: phosphatebuffered saline; $\mathrm{PGE}_{2}$ : prostaglandin $\mathrm{E}_{2}$; PMSF: phenylmethylsulphonyl fluoride; SDS-PAGE: sodium dodecyl sulphate-polyacrylamide gel electrophoresis; TNF- $\alpha$ : tumor necrosis factor alpha. 


\section{Acknowledgements}

The authors' work was supported in part by grants from National Science Council (NSC 96-2314-B-016-015-MY3; NSC 96-2314-B-400-003-MY2 and NSC 98-2314-B-400-002-MY3) and from Tri-Service General Hospital (TSGH-C98-08S02),Taiwan, ROC. The authors are also grateful to Dr S.-F. Yang for her kind gift of GST-c-Jun fusion protein.

\section{Author details}

${ }^{1}$ Graduate Institute of Medical Science, National Defense Medical Center, Neihu 114, Taipei, Taiwan, ROC. ${ }^{2}$ Division of Rheumatology/lmmunology \& Allergy, Department of Medicine, Tri-Service General Hospital, National Defense Medical Center, Neihu 114, Taipei, Taiwan, ROC. ${ }^{3}$ Institute of Cellular and System Medicine, National Health Research Institute, Zhunan, Miaoli County 350, Taiwan, ROC. ${ }^{4}$ Graduate Institute of Basic Medical Science, PhD Program of Aging, China Medical University, Taichung 40402, Taiwan, ROC.

\section{Authors' contributions}

FCL designed the study, performed most of the experiments, wrote the manuscript and took responsibility for the correctness of the study results. $J H L$ and $L H$ critically corrected the manuscript, gave advice and guided experimental steps along the experimental process. WLW and DMC helped to perform part of the cartilage explants cultures, histological analysis, proteoglycan measurements and reviewed the manuscript. All authors read and approved the final manuscript.

\section{Competing interests}

The authors declare that they have no competing interests.

Received: 18 May 2010 Revised: 28 July 2010

Accepted: 8 September 2010 Published: 8 September 2010

\section{References}

1. Herrero-Beaumont G, Roman-Blas JA, Castaneda S, Jimenez SA: Primary osteoarthritis no longer primary: three subsets with distinct etiological, clinical, and therapeutic characteristics. Semin Arthritis Rheum 2009, 39:71-80.

2. Abramson SB, Attur M: Developments in the scientific understanding of osteoarthritis. Arthritis Res Ther 2009, 11:227.

3. Loeser RF, Yammani RR, Carlson CS, Chen H, Cole A, Im HJ, Bursch LS, Yan SD: Articular chondrocytes express the receptor for advanced glycation end products: Potential role in osteoarthritis. Arthritis Rheum 2005, 52:2376-2385.

4. Pisetsky DS, Erlandsson-Harris $\mathrm{H}$, Andersson U: High-mobility group box protein 1 (HMGB1): an alarmin mediating the pathogenesis of rheumatic disease. Arthritis Res Ther 2008, 10:209.

5. Verzijl N, DeGroot J, Ben ZC, Brau-Benjamin O, Maroudas A, Bank RA, Mizrahi J, Schalkwijk CG, Thorpe SR, Baynes JW, Bijlsma JW, Lafeber FP, TeKoppele JM: Crosslinking by advanced glycation end products increases the stiffness of the collagen network in human articular cartilage: a possible mechanism through which age is a risk factor for osteoarthritis. Arthritis Rheum 2002, 46:114-123.

6. Yammani RR, Carlson CS, Bresnick AR, Loeser RF: Increase in production of matrix metalloproteinase 13 by human articular chondrocytes due to stimulation with S100A4: Role of the receptor for advanced glycation end products. Arthritis Rheum 2006, 54:2901-2911.

7. Yan SF, Ramasamy R, Schmidt AM: Soluble RAGE: therapy and biomarker in unraveling the RAGE axis in chronic disease and aging. Biochem Pharmacol 2010, 79:1379-1386.

8. Brisdelli F, D'Andrea G, Bozzi A: Resveratrol: a natural polyphenol with multiple chemopreventive properties. Curr Drug Metab 2009, 10:530-546.

9. Penumathsa SV, Maulik N: Resveratrol: a promising agent in promoting cardioprotection against coronary heart disease. Can I Physiol Pharmacol 2009, 87:275-286.

10. Mainardi T, Kapoor S, Bielory L: Complementary and alternative medicine: herbs, phytochemicals and vitamins and their immunologic effects. J Allergy Clin Immunol 2009, 123:283-294, quiz 295-296.

11. Elmali N, Esenkaya I, Harma A, Ertem K, Turkoz Y, Mizrak B: Effect of resveratrol in experimental osteoarthritis in rabbits. Inflamm Res 2005, 54:158-162.

12. Shakibaei M, Csaki C, Nebrich S, Mobasheri A: Resveratrol suppresses interleukin-1 beta-induced inflammatory signaling and apoptosis in human articular chondrocytes: potential for use as a novel nutraceutical for the treatment of osteoarthritis. Biochem Pharmacol 2008, 76:1426-1439.

13. Dave M, Attur M, Palmer G, Al-Mussawir HE, Kennish L, Patel J, Abramson SB: The antioxidant resveratrol protects against chondrocyte apoptosis via effects on mitochondrial polarization and ATP production. Arthritis Rheum 2008, 58:2786-2797.

14. Csaki C, Keshishzadeh N, Fischer K, Shakibaei M: Regulation of inflammation signalling by resveratrol in human chondrocytes in vitro. Biochem Pharmacol 2008, 75:677-687.

15. Pelletier JP, Martel-Pelletier J, Abramson SB: Osteoarthritis, an inflammatory disease: potential implication for the selection of new therapeutic targets. Arthritis Rheum 2001, 44:1237-1247.

16. Nah SS, Choi IY, Lee CK, Oh JS, Kim YG, Moon HB, Yoo B: Effects of advanced glycation end products on the expression of COX-2, PGE2 and NO in human osteoarthritic chondrocytes. Rheumatology (Oxford) 2008, 47:425-431.

17. Huang CY, Hung LF, Liang CC, Ho LJ: COX-2 and iNOS are critical in advanced glycation end product-activated chondrocytes in vitro. Eur J Clin Invest 2009, 39:417-428.

18. Ahmed MU, Brinkmann Frye E, Degenhardt TP, Thorpe SR, Baynes JW: Nepsilon-(carboxyethyl)lysine, a product of the chemical modification of proteins by methylglyoxal, increases with age in human lens proteins. Biochem J 1997, 324:565-570.

19. Farboud B, Aotaki-Keen A, Miyata T, Hjelmeland LM, Handa JT: Development of a polyclonal antibody with broad epitope specificity for advanced glycation endproducts and localization of these epitopes in Bruch's membrane of the aging eye. Mol Vis 1999, 5:11.

20. Rasheed Z, Anbazhagan AN, Akhtar N, Ramamurthy S, Voss FR, Haqqi TM: Green tea polyphenol epigallocatechin-3-gallate inhibits advanced glycation end product-induced expression of tumor necrosis factoralpha and matrix metalloproteinase-13 in human chondrocytes. Arthritis Res Ther 2009, 11:R71.

21. Lai JH, Ho L, Kwan CY, Chang DM, Lee TC: Plant alkaloid tetrandrine and its analog block CD28-costimulated activities of human peripheral blood T cells: potential immunosuppressants in transplantation immunology. Transplantation 1999, 68:1383-1392.

22. Ho LJ, Lin LC, Hung LF, Wang SJ, Lee CH, Chang DM, Lai JH, Tai TY: Retinoic acid blocks pro-inflammatory cytokine-induced matrix metalloproteinase production by down-regulating JNK-AP-1 signaling in human chondrocytes. Biochem Pharmacol 2005, 70:200-208.

23. Gupta K, Shukla M, Cowland JB, Malemud CJ, Haqqi TM: Neutrophil gelatinase-associated lipocalin is expressed in osteoarthritis and forms a complex with matrix metalloproteinase 9. Arthritis Rheum 2007, 56:3326-3335.

24. Schmidt-Hansen B, Ornas D, Grigorian M, Klingelhofer J, Tulchinsky E, Lukanidin E, Ambartsumian N: Extracellular S100A4(mts1) stimulates invasive growth of mouse endothelial cells and modulates MMP-13 matrix metalloproteinase activity. Oncogene 2004, 23:5487-5495.

25. Upton ML, Chen J, Setton LA: Region-specific constitutive gene expression in the adult porcine meniscus. J Orthop Res 2006, 24:1562-1570.

26. Chou CH, Cheng WT, Kuo TF, Sun JS, Lin FH, Tsai JC: Fibrin glue mixed with gelatin/hyaluronic acid/chondroitin-6-sulfate tri-copolymer for articular cartilage tissue engineering: the results of real-time polymerase chain reaction. J Biomed Mater Res A 2007, 82:757-767.

27. van Lent PL, Grevers LC, Blom AB, Arntz OJ, van de Loo FA, van der Kraan P, Abdollahi-Roodsaz S, Srikrishna G, Freeze H, Sloetjes A, Nacken W, Vogl T, Roth J, van den Berg WB: Stimulation of chondrocyte-mediated cartilage destruction by S100A8 in experimental murine arthritis. Arthritis Rheum 2008, 58:3776-3787.

28. Allport VC, Slater DM, Newton R, Bennett PR: NF-kappaB and AP-1 are required for cyclo-oxygenase 2 gene expression in amnion epithelial cell line (WISH). Mol Hum Reprod 2000, 6:561-565.

29. Cho MK, Suh SH, Kim SG: JunB/AP-1 and NF-kappa B-mediated induction of nitric oxide synthase by bovine type I collagen in serum-stimulated murine macrophages. Nitric Oxide 2002, 6:319-332.

30. Mengshol JA, Vincenti MP, Brinckerhoff CE: IL-1 induces collagenase-3 (MMP-13) promoter activity in stably transfected chondrocytic cells: requirement for Runx-2 and activation by p38 MAPK and JNK pathways. Nucleic Acids Res 2001, 29:4361-4372. 
31. Verzijl N, DeGroot J, Oldehinkel E, Bank RA, Thorpe SR, Baynes JW Bayliss MT, Bijlsma JW, Lafeber FP, Tekoppele JM: Age-related accumulation of Maillard reaction products in human articular cartilage collagen. Biochem J 2000, 350:381-387.

32. DeGroot J, Verzijl N, Bank RA, Lafeber FP, Bijlsma JW, Tekoppele JM: Agerelated decrease in proteoglycan synthesis of human articular chondrocytes: the role of nonenzymatic glycation. Arthritis Rheum 1999, 42:1003-1009.

33. DeGroot J, Verzijl N, Wenting-van Wijk MJ, Jacobs KM, Van El B, Van Roermund PM, Bank RA, Bijlsma JW, TeKoppele JM, Lafeber FP: Accumulation of advanced glycation end products as a molecular mechanism for aging as a risk factor in osteoarthritis. Arthritis Rheum 2004, 50:1207-1215.

34. Handl M, Filova E, Kubala M, Lansky Z, Kolacna L, Vorlicek J, Trc T, Pach M, Amler E: Fluorescent advanced glycation end products in the detection of factual stages of cartilage degeneration. Physiol Res 2007, 56:235-242.

35. Amin AR, Attur M, Abramson SB: Nitric oxide synthase and cyclooxygenases: distribution, regulation, and intervention in arthritis. Curr Opin Rheumatol 1999, 11:202-209.

36. Abramson SB, Attur $M$, Amin AR, Clancy R: Nitric oxide and inflammatory mediators in the perpetuation of osteoarthritis. Curr Rheumatol Rep 2001, 3:535-541.

37. Pelletier JP, Jovanovic D, Fernandes JC, Manning P, Connor JR, Currie MG, Di Battista JA, Martel-Pelletier J: Reduced progression of experimental osteoarthritis in vivo by selective inhibition of inducible nitric oxide synthase. Arthritis Rheum 1998, 41:1275-1286.

38. Pelletier JP, Jovanovic DV, Lascau-Coman V, Fernandes JC, Manning PT, Connor JR, Currie MG, Martel-Pelletier J: Selective inhibition of inducible nitric oxide synthase reduces progression of experimental osteoarthritis in vivo: possible link with the reduction in chondrocyte apoptosis and caspase 3 level. Arthritis Rheum 2000, 43:1290-1299.

39. Pelletier JP, Fernandes JC, Jovanovic DV, Reboul P, Martel-Pelletier J: Chondrocyte death in experimental osteoarthritis is mediated by MEK $1 /$ 2 and p38 pathways: role of cyclooxygenase- 2 and inducible nitric oxide synthase. J Rheumatol 2001, 28:2509-2519.

40. Sadowski T, Steinmeyer J: Effects of non-steroidal antiinflammatory drugs and dexamethasone on the activity and expression of matrix metalloproteinase-1, matrix metalloproteinase-3 and tissue inhibitor of metalloproteinases- 1 by bovine articular chondrocytes. Osteoarthritis Cartilage 2001, 9:407-415.

41. Gaestel M, Kotlyarov A, Kracht M: Targeting innate immunity protein kinase signalling in inflammation. Nat Rev Drug Discov 2009, 8:480-499.

42. Mitchell PG, Magna HA, Reeves LM, Lopresti-Morrow LL, Yocum SA, Rosner PJ, Geoghegan KF, Hambor JE: Cloning, expression, and type II collagenolytic activity of matrix metalloproteinase-13 from human osteoarthritic cartilage. J Clin Invest 1996, 97:761-768.

43. Neuhold LA, Killar L, Zhao W, Sung ML, Warner L, Kulik J, Turner J, Wu W, Billinghurst C, Meijers T, Poole AR, Babij P, DeGennaro LJ: Postnatal expression in hyaline cartilage of constitutively active human collagenase-3 (MMP-13) induces osteoarthritis in mice. J Clin Invest 2001, 107:35-44.

44. Burrage PS, Mix KS, Brinckerhoff CE: Matrix metalloproteinases: role in arthritis. Front Biosci 2006, 11:529-543.

45. Miwa HE, Gerken TA, Hering TM: Effects of covalently attached chondroitin sulfate on aggrecan cleavage by ADAMTS-4 and MMP-13. Matrix Biol 2006, 25:534-545.

46. Wallerath T, Deckert G, Ternes T, Anderson H, Li H, Witte K, Forstermann U: Resveratrol, a polyphenolic phytoalexin present in red wine, enhances expression and activity of endothelial nitric oxide synthase. Circulation 2002, 106:1652-1658.

47. Bertelli AA, Giovannini L, Stradi R, Urien S, Tillement JP, Bertelli A: Evaluation of kinetic parameters of natural phytoalexin in resveratrol orally administered in wine to rats. Drugs Exp Clin Res 1998, 24:51-55.

48. Bertelli A, Bertelli AA, Gozzini A, Giovannini L: Plasma and tissue resveratrol concentrations and pharmacological activity. Drugs Exp Clin Res 1998, 24:133-138.

49. Tan Y, Lim LH: trans-Resveratrol, an extract of red wine, inhibits human eosinophil activation and degranulation. Br J Pharmacol 2008, 155:995-1004.

\section{doi:10.1186/ar3127}

Cite this article as: Liu et al: Chondroprotective effects and mechanisms of resveratrol in advanced glycation end productsstimulated chondrocytes. Arthritis Research \& Therapy 2010 12:R167.

\section{Submit your next manuscript to BioMed Central and take full advantage of:}

- Convenient online submission

- Thorough peer review

- No space constraints or color figure charges

- Immediate publication on acceptance

- Inclusion in PubMed, CAS, Scopus and Google Scholar

- Research which is freely available for redistribution

Submit your manuscript at www.biomedcentral.com/submit
Ciomed Central 